\title{
Clinical presentation, natural history, and cumulative death rates of 230 adults with primary cryptococcal meningitis in Zambian AIDS patients treated under local conditions
}

\author{
P Mwaba, J Mwansa, C Chintu, J Pobee, M Scarborough, S Portsmouth, A Zumla
}

Department of Medicine and the UNZA-UCLMS Project, University Teaching Hospital, Lusaka, Zambia P Mwaba

Department of Pathology and Microbiology, University Teaching Hospital, Lusaka, Zambia

J Mwansa

UNZA-UCLMS Project, University Teaching Hospital, Lusaka, Zambia

C Chintu

Department of Medicine, University Teaching Hospital, Lusaka, Zambia J Pobee

University College London Hospitals Trust, London, UK M Scarborough $S$ Portsmouth

UNZA-UCLMS Project, University Teaching Hospital, Lusaka, Zambia and Centre for Infectious Diseases and International Health, Royal Free and University College Medical School, University College London, University College London Hospitals Trust, London, UK

A Zumla

Correspondence to: Professor Alimuddin Zumla, Centre for Infectious Diseases and International Health, University College London, Windeyer Institute, Room G41, 46 Cleveland Street, London W1P 6DB, UK

a.zumla@ucl.ac.uk

Submitted 24 April 2001 Accepted 14 June 2001
Abstract

Setting-Inpatient medical wards, Department of Medicine, University Teaching Hospital, Lusaka, Zambia.

Objective-To define the natural history, clinical presentation, and management outcome of microbiologically confirmed cryptococcal meningitis in adult AIDS patients treated under local conditions where antifungal and antiretroviral therapies are not routinely available.

Design-A descriptive, longitudinal, observational study.

Methods-All adult patients admitted to the medical wards of the University Teaching Hospital, Lusaka, Zambia with cerebrospinal fluid culture proved, primary cryptococcal meningitis, during a 12 month period were enrolled into the study. The following details were acquired: clinical features, HIV status, laboratory data, treatment accorded, and survival.

Results-A total of 230 patients with primary cryptococcal meningitis were studied (median age 32 years; range 15-65 years; 112 males, 118 females). Cryptococcal meningitis was the first AIDS defining illness in $210(91 \%)$ patients. One hundred and thirty of the $230(56 \%)$ patients had received treatment with fluconazole monotherapy and $100(43 \%)$ patients received palliative care only without any antifungal therapy. A $\mathbf{1 0 0 \%}$ case fatality rate was observed in both groups at follow up: by seven weeks in the untreated group and at six months in the fluconazole treated group. The cumulative median survival from time of diagnosis was 19 days (range 1-164 days) for the fluconazole treated group and 10 days (range 0-42 days) for the untreated group.

Conclusion-Cryptococcal meningitis, under current treatment accorded at the University Teaching Hospital, Lusaka, has a $100 \%$ mortality in young Zambian adults with AIDS. The current treatment accorded to Zambian adults with cryptococcal meningitis is inappropriate. An urgent need exists to improve strategies for the clinical management of AIDS patients in poor African countries. The wider ethical and operational issues of making available antifungals to African AIDS patients are discussed.

(Postgrad Med f 2001;77:769-773)
Keywords: cryptococcal meningitis; Africa; AIDS/HIV; natural history

Cryptococcal meningitis, caused by the environmental encapsulated fungus Cryptococcus neoformans, has become the most common lethal fungal infection in patients with AIDS worldwide. ${ }^{1-7}$ Effective antifungal therapy followed by lifelong prophylaxis with fluconazole, coupled with highly active antiretroviral therapy has greatly reduced morbidity and mortality in AIDS patients with cryptococcal infection in the USA and Europe. However, longitudinal studies evaluating the treatment outcomes of patients with cryptococcal meningitis from sub-Saharan Africa, where the predominant global burden of HIV infection occurs, are scant.

In Zambia clinical observations indicate that cryptococcal meningitis is now the commonest cause of meningitis in adults patients presenting to the University Teaching Hospital in Lusaka. The Zambian Ministry of Health has a list of essential drugs that are supplied to all health institutions in the country for the management of HIV/AIDS related opportunistic infections and non-opportunistic conditions. Due to economic constraints and cost-benefit considerations, antifungal and antiretroviral drugs are not supplied by the Ministry of Health and thus the majority of patients who cannot afford to pay for these drugs go without any treatment. Fluconazole monotherapy was the recommended treatment regimen at the time of the study ( $400 \mathrm{mg}$ immediate dose followed by $200 \mathrm{mg}$ daily continued indefinitely). Stocks of this drug at the University Teaching Hospital in Lusaka depend on donations from private donors and drug companies. Clinical observations indicate that treatment of HIV infected patients and their associated complications such as cryptococcal meningitis at the University Teaching Hospital is unrewarding and ineffectual. This study was undertaken to obtain information on the clinical presentation, natural history, and cumulative death rates of cryptococcal meningitis in adult Zambian AIDS patients treated under local conditions.

Patients and methods

The University Teaching Hospital, which serves the population ( 1.1 million) of Lusaka, is a tertiary care referral centre, although 
Table 1 Presenting symptoms of 230 cases of primary cryptococcal meningitis

\begin{tabular}{ll}
\hline Symptom & No (\%) \\
\hline Headache & $209(91)$ \\
Weight loss & $208(90)$ \\
Fever & $120(52)$ \\
Forgetfulness & $114(50)$ \\
Slowness & $110(48)$ \\
Pruritis/rash & $92(40)$ \\
Vomiting & $85(37)$ \\
Social withdrawal & $36(16)$ \\
Confusion & $29(13)$ \\
Diarrhoea & $22(10)$ \\
Fits & $20(9)$ \\
Paralysis & $6(3)$ \\
\hline
\end{tabular}

Table 2 Presenting clinical signs of 230 cases of primary cryptococcal meningitis

\begin{tabular}{ll}
\hline Sign & No (\%) \\
\hline Fever & $210(91)$ \\
Wasting & $206(90)$ \\
Meningeal signs & $195(85)$ \\
Anaemia & $161(70)$ \\
Oral candida & $132(57)$ \\
Rash & $131(57)$ \\
Lymphadenopathy & $109(47)$ \\
Motor weakness & $93(40)$ \\
Malnutrition & $82(36)$ \\
Scar of herpes zoster & $45(20)$ \\
Papilloedema & $44(19)$ \\
Confusion & $31(13)$ \\
Cranial nerve palsy & $29(13)$ \\
\hline
\end{tabular}

patients have direct access to services via accident and emergency. All adult patients (over the age of 15 years) admitted to the Department of Medicine adult inpatient wards of the University Teaching Hospital, over a period of 12 months (15 January 1998 to 14 January 1999), with cerebrospinal fluid (CSF) culture proved primary cryptococcal meningitis were enrolled into the study. Informed consent was obtained from the patients or their immediate relatives when the diagnosis had been confirmed. Patients' socioeconomic status, residential address, symptoms and signs, and evidence of other AIDS defining illnesses were recorded on the enrolment form. At the time of the study, blood cultures for bacteria and lumbar punctures were routinely performed on all patients at admission. CSF was subjected to microscopy, and Gram, alcohol and acid-fast bacilli, and Indian ink staining. Cryptococcal antigen test on CSF was not performed as routine because of intermittent reagent supply. Thus, for the purpose of this study, only those patients with CSF culture positive cryptococcal meningitis were enrolled and thus patients with less florid cryptococcal meningitis may have been excluded by this stringent criteria. All cryptococcal isolates were subcultured onto Sabouraud dextrose and blood agar media. Full blood counts, erythrocyte sedimentation rate, malarial parasite films, and chest radiography were performed routinely at admission.

Table 3 CSF findings of 230 patients with cryptococcal meningitis; values are range (mean)

\begin{tabular}{llll}
\hline & Proteins $(\mathrm{mg} / \mathrm{l})$ & Glucose $($ mmol/l) & Leucocytes $/ \mathrm{ml}$ \\
\hline Treated group & $356-3000(1039)$ & $0.2-2.8(1.31)$ & $0-25(9)$ \\
Untreated group & $358-3000(1017)$ & $0.23-2.78(1.33)$ & $0-22(10)$ \\
\hline
\end{tabular}

HIV-1 serology was performed using two enzyme linked immunoassays (ELISA) tests (Welcozyme, Wellcome diagnostics, Dartford, Oxford, UK) and an antiglobulin recombinant ELISA (DuPont Nemoirs, Delaware, USA). Sera testing positive by one or other were subjected to western blot. HIV-2 has not been reported to be of significance in Zambia. The treatment accorded was noted. Due to limited pharmacy stocks, only patients who were admitted in the first half of the year received the locally recommended treatment for cryptococcal meningitis: one immediate dose of fluconazole $400 \mathrm{mg}$, intravenous or oral depending on clinical state, and fluconazole $200 \mathrm{mg}$ orally daily thereafter. Due to pressure of bed space, hospital policy is to get the patients home as soon as possible and management is shared with the palliative care teams. Those patients who were discharged from hospital were given a six month supply of fluconazole (three months with patient and three months with palliative care team) and were followed up by home based care teams. Patients were reviewed at home or at hospital outpatient clinics. Follow up visits occurred at two, six, nine, 12, 16, 20, and 24 weeks after discharge. Where patients died in between visits, the date of death was obtained from next of kin and recorded. No postmortem examinations were performed.

ETHICAL APPROVAL

This study was approved by the University of Zambia Research and Ethics Committee.

STATISTICAL ANALYSIS

This was performed using EpiInfo version 6.03 . The cumulative median survival was calculated from the date of presentation by using the Kaplan-Meier method.

\section{Results}

Over the period of 12 months, 230 patients with CSF culture proved primary cryptococcal meningitis were enrolled into the study, all of whom were HIV positive. Of these, 210 cases (91\%) had cryptococcal meningitis as the first AIDS defining illness. The median age was 32 years; age range 15 to 65 years and 112 were males and 118 females. Clinical features and CSF laboratory findings are detailed in tables 1,2 , and 3 .

While a wide range of symptoms and signs occurred, those localising to the central nervous system were dominant. Weight loss was a constant feature of most patients. Due to (donated) stocks of fluconazole running out at the University Teaching Hospital during the first half of the year of study, only 130 patients received treatment with fluconazole monotherapy and 100 patients received only palliative care. Patients were hospitalised for a median of four days (range 0-21 days). The cumulative median survival from time of diagnosis was 19 days (range 1-168 days) for the fluconazole group and 10 days (95\% range 0-42 days) for the untreated group. 
Table 4 Cumulative survival rates of Zambian patients with cryptococcal meningitis

\begin{tabular}{lll}
\hline $\begin{array}{l}\text { Time from } \\
\text { diagnosis (days) }\end{array}$ & $\begin{array}{l}\text { No (\%) of fluconazole } \\
\text { treated patients } \\
\text { surviving }(n=130)\end{array}$ & $\begin{array}{l}\text { No (\%) of untreated } \\
\text { patients surviving } \\
(n=100)\end{array}$ \\
\hline 7 & $101(78)$ & $64(64)$ \\
14 & $80(61)$ & $35(35)$ \\
21 & $26(20)$ & $12(12)$ \\
42 & $13(10)$ & 0 \\
84 & $5(4)$ & 0 \\
126 & $3(2)$ & 0 \\
168 & 0 & 0 \\
\hline
\end{tabular}

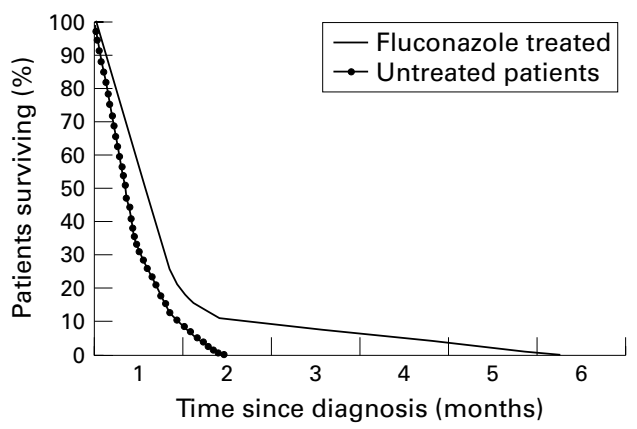

Figure 1 Kaplan-Meier survival curves for 130 patients treated with fluconazole and 100 patients who received no antifungal treatment.

Cumulative death rates are shown in table 4 and in fig 1 . Overall, the mortality of primary cryptococcal meningitis was $100 \%$ at six months of follow up. Those patients who did not receive antifungal treatment had all died by the end of week 7 and those who received treatment with fluconazole had all died by week 24 . There were no significant differences in clinical features between the fluconazole treated and untreated groups, demographic features, educational level attained, marital status, age and sex distribution, and laboratory findings (data not shown) between those who received fluconazole therapy and those who did not.

\section{Discussion}

Before the introduction of the antifungal amphotericin B therapy in the mid-1950s for cryptococcal meningitis, the mortality rate was $100 \%$ for these cases. Published studies from the USA and Europe indicate that while current treatment regimens are still associated with acute mortality rates during initial therapy, the 12 month survival rates among all patients with maintenance therapy are significantly improved and a large proportion of patients do extremely well on maintenance antifungal therapy. ${ }^{4-68}$ The introduction of antiretroviral treatment regimens has further improved morbidity and mortality from fungal infections in AIDS patients in the west.

Our observational study of cryptococcal meningitis in AIDS patients, one of the largest so far from Central Africa, provides further insight into the clinical presentation, natural history, and outcome of this disease in the subSaharan African situation. The results of this study substantiates our clinical observations over the past decade at the University Teaching
Hospital in Lusaka of an inevitable 100\% mortality associated with cryptococcal meningitis in patients with AIDS. In our study patients receiving fluconazole therapy did not show a significant survival advantage. The cumulative median survival was 10 days in those patients who received no antifungal therapy and 19 days in those who did. Case definition for this study restricted entry to those patients who had cryptococci isolated in culture from CSF. This may have biased the study to those patients who had more serious illness. Due to a restricted budget, the University Teaching Hospital does not perform CD4+ lymphocyte counts nor HIV viral load measurements on any of the patients and thus we were not able to determine the degree of immunosuppression in each patient.

All patients who were started on fluconazole had adequate stocks of fluconazole to last at least six months and thus drug shortage would not account for the lack of survival advantage. Non-compliance may have contributed to treatment failures but regular follow up indicated that patients were keen to take therapy. Fluconazole monotherapy is not the optimal treatment for cryptococcal meningitis, ${ }^{46810}$ and although the local fluconazole resistance patterns of Cryptoccocus spp are not known, this may be the prime reason for the $100 \%$ mortality seen at six months of follow up. Studies examining fluconazole monotherapy have shown that doses higher than those used at the University Teaching Hospital in Lusaka may have some improved effect. ${ }^{112}$ The dose of fluconazole used in patients studied was probably subtherapeutic. At the correct dose, fluconazole is only marginally less efficient than amphotericin B. A $400 \mathrm{mg} /$ day prophylactic dose has been shown to be more effective than $200 \mathrm{mg} /$ day. The results of this study were used to persuade the authorities to introduce a more uniform policy for the treatment of cryptococcal meningitis at the University Teaching Hospital, Lusaka. The current regimen includes use of amphotericin B initially with a maintenance dose of fluconazole at $400 \mathrm{mg}$ daily. Financial constraints throughout most resource poor countries prohibit the widespread use of high dose fluconazole regimens or drugs such as amphotericin B. It is possible that the addition of flucytosine to fluconazole may produce improved results in these patients, as shown in a Ugandan study, ${ }^{2}$ although this adds additional cost pressures to an already overstretched health system.

Reports from neighbouring African countries indicate that this appalling statistic for cryptococcal meningitis in AIDS patients is a regional problem, attributable to inability of the health services to provide appropriate health care due to the economic difficulties facing many sub-Saharan African countries. A study from neighbouring Malawi identified 31 patients from laboratory records. ${ }^{14}$ Only six patients received treatment, four with fluconazole, and two with amphotericin B (of which one had maintenance fluconazole after induction therapy). Though the majority were probably HIV positive this was not specifically 
tested. In a four year study from Durban 65 patients with culture positive cryptococcal meningitis were assessed retrospectively. ${ }^{3}$ Sixty eight per cent (44) were coinfected with HIV. Though most patients would have received amphotericin B (+/- flucytosine) or fluconazole this was poorly recorded and therefore uninterpretable. In a smaller study of 89 patients from Zimbabwe, no specific antifungal treatment was given, and the cumulative median survival after diagnosis was 14 days (range $0-233$ days) with $22 \%$ of patients surviving more than 30 days. ${ }^{1}$

Our study has shown that cryptococcal meningitis is a common AIDS defining illness in young Zambian adults and is associated with an invariably fatal outcome. At the University Teaching Hospital in Lusaka cryptococcal meningitis is the fourth commonest diagnosis requiring admission to the medical wards after community acquired pneumonia, tuberculosis, and diarrhoea and wasting. As described by other studies from Africa, ${ }^{1-3} 14$ the majority of our patients were young adults of both sexes, who also suffered from other potentially lethal opportunistic infections. Current economic constraints on health services cause regular shortages of commonly used antibiotics and antituberculosis drugs, and thus palliative care will remain the mainstay of treatment for AIDS patients with cryptococcal meningitis. In Zambia, HIV infection is widespread and HIV seroprevalence rates are among the highest in the world. Recent serosurveys show that 36\% of antenatal Zambian women are HIV positive. ${ }^{15}$ This phenomenal increase in HIV infection is paralleled by similar figures being observed in neighbouring countries. The unavailability of antiretroviral and antifungal agents to the majority of the populations of these countries means a continued increase in the number of deaths from cryptococcal meningitis in the next few years is likely. Cryptococcal meningitis is just one of many opportunistic infections complicating the lives of HIV infected Africans. Its invariably fatal outcome, as demonstrated by this study, highlights the need for concerted action to make available essential drugs for the prevention and treatment of these disorders. People in developing countries worldwide have virtually no access to new essential drugs, which are priced beyond their means. This raises several major ethical issues, not least being contravention of the right to a standard of living adequate for health as enshrined in Article 25 of the United Nations Universal Declaration of Human Rights. ${ }^{16}$

The national essential drugs programme in Zambia aims to ensure both an equitable and rational use of limited resources. Many drugs considered essential to the management of HIV infection in the West are not affordable. Relying on donations of essential drugs is unsustainable and liable to intermittent supply problems. Irrespective of the survival outcomes in this instance, only $56 \%$ of patients had access to the recommended drug. This places medical staff in an untenable position and reduces the patient's experience to little more than a treatment lottery. Reducing costs is just one element of a strategy needed to make essential drugs more accessible. Health systems also need the capacity to deliver effective treatment and support people living with HIV/ AIDS. The cost of effective drugs is, however, a major force driving increasing inequality of access to treatment between industrialised and developing countries.

In Zambia, with an average income of one US dollar or less per day, the poorest quarter of the population cannot afford even the most basic requisites for healthy living, let alone buy antiretroviral or antifungal agents. Governments in the developing world are limited in their ability to provide basic health care, partly as a result of their requirement to pay interest on loans from the wealthy nations. In Zambia, for example, for every dollar spent on health care, four dollars are spent on servicing international debt. While African governments battle with repayment of multibillion pound debts, there are signs of continued deterioration of health care delivery. ${ }^{17}$

As the incidence of AIDS in Africa increases, the need for uniform, rational, and sustainable approaches towards the overall management of AIDS patients become more urgent. The staggering mortality from infectious diseases for which effective treatments are available in the West is a direct result of poverty consequential upon an unjust social and economic order. The current debate on costs and production of antiretroviral drugs should be extended to the cost and availability of drugs for the treatment of cryptococcal meningitis. Until further information is available on the relative efficacy of different treatment regimens, management will continue to be ineffective even if antifungal drugs became available and affordable.

We are grateful to the Health and Population Department of the UK Department for International Development for their support. Dr Peter Mwaba received support from the Beit Trust.

1 Heyderman RS, Gangaidzo IT, Hakim JG, et al. Cryptococcal meningitis in human immunodeficiency virus infected patients in Harare, Zimbabwe. Clin Infect Dis 1998;26:284-

2 Mayanja-Kizza H, Oishi K, Mitarai S, et al. Combination therapy with fluconazole and flucytosine for cryptococcal 1998;26:1362-6.

3 Moosa MYS, Coovadia YM. Cryptococcal menigitis in Durban, South Africa: a comparison of clinical features, laboratory findings, and outcome for human immunodeficiency virus (HIV)-positive and HIV-negative patients.

4 Powderly WG. Recent advances in the management of cryptococcal meningitis in patients with AIDS. Clin Infect Dis 1996;22(suppl 2):S119-23.

5 Sharkey PK, Graybill JR, Johnson ES, et al. Amphotericin B lipid complex compared with amphotericin B in the treatment of cryptococcal meningitis in patients with AIDS. Clin Infect Dis 1996;22:315-21.

6 Van der Horst CM, Saag MS, Cloud GA, et al. Treatment of cryptococcal meningitis associated with the acquired immunodeficiency syndrome. N Engl f Med 1997;337:1521.

7 Khanna N, Chandramuki A, Desai A, et al. Cryptococcal infections of the central nervous system: an analysis of predisposing factors, laboratory findings and outcome in patients from South India with special reference to HIV

8 De-Lalla F, Pellizzer G, Vagia A, et al. Amphotericin B as primary therapy for cryptococcosis in patients with AIDS: primary therapy for cryptococcosis in patients with AIDS: relatively short period. Clin Infect Dis 1995;20:263-6. 
9 Anthony SJ, Patel A, Leonard J. Use of fluconazole in the treatment of non-AIDS cryptococcal meningitis. F Natl Med 1997;89:694-5.

10 Leenders AC, Reiss P, Portegies P, et al. Liposomal amphotericin B (AmBisome) compared with amphotericin B both followed by oral fluconazole in the treatment of AIDS associated cryptococcal meningitis. AIDS 1997;11:1463-71.

11 Haubrich RH, Haghighat D, Bozzette SA, et al. High dose fluconazole for treatment of cryptococcal disease in patients with human immunodeficiency virus infection. F Infect Dis 1994;170:238-42.

12 Menichetti F, Fiorio M, Tosti A, et al. High dose fluconazole therapy for cryptococcal meningitis in patients with AIDS. Clin Infect Dis 1996;22:838-40.
13 Aberg JA, Powderly WG. Cryptococcal disease: implications of recent clinical trials on treatment and management. AIDS Clin Rev 1997;98:229-48.

14 Maher D, Mwandumba H. Cryptococcal meningitis in Lilongwe and Blantyre, Malawi. F Infect 1994;28:59-64.

15 Fylkesnes K, Musonda RM, Kasumba K, et al. The HIV epidemic in Zambia: socio-demographic prevalence patterns and indications of trends among childbearing women. AIDS 1997;11:339-45.

16 United Nations. Universal declaration of human rights. New York: United Nations, 1948.

17 Jubilee 2000. A debt cutter's handbook. London: Jubilee, 2000: 1996.

\section{7th European Forum on Quality Improvement in Health Care}

\section{1-23 March 2002 \\ Edinburgh, Scotland}

We are delighted to announce this forthcoming conference in Edinburgh.

The themes of the Forum are:

- Leadership, culture change, and change management

- Achieving radical improvement by redesigning care

- Health policy for lasting improvement in health care systems

- Patient safety

- Measurement for improvement, learning, and accountability

- Partnership with patients

- Professional quality: the foundation for improvement

- Continuous improvement in education and training

- People and improvement.

Presented to you by the BMJ Publishing Group (London, UK) and Institute for Healthcare Improvement (Boston, USA). For more information contact: quality@bma.org.uk or look at the website www.quality.bmjpg.com. Tel: +44 (0)20 7383 6409; fax: +44 (0)20 73736869 . 